IOS Press

\title{
Administration of cultured autologous bone marrow stromal cells into cerebrospinal fluid in spinal injury patients: A pilot study
}

\author{
Fukuki Saito $^{\mathrm{a}}$, Toshio Nakatani ${ }^{\mathrm{a}, *}$, Masaaki Iwase ${ }^{\mathrm{a}}$, Yuji Maeda ${ }^{\mathrm{a}}$, Yoshinori Murao ${ }^{\mathrm{a}}$, \\ Yoshihisa Suzuki ${ }^{\mathrm{b}}$, Masanori Fukushima ${ }^{\mathrm{c}}$ and Chizuka Ide ${ }^{\mathrm{d}}$ \\ ${ }^{a}$ Emergency and Critical Care Center, Kansai Medical University, Moriguchi, Osaka, Japan \\ ${ }^{\mathrm{b}}$ Department of Plastic and Reconstructive Surgery, Kitano Hospital, Tazuke Kofukai Medical Research Institute, \\ Osaka, Japan \\ ${ }^{\mathrm{c}}$ Translational Research Informatics Center, Kobe, Japan \\ ${ }^{\mathrm{d}}$ Institute of Regeneration and Rehabilitation, Faculty of Nursing and Rehabilitation, Aino University, \\ Osaka, Japan
}

\begin{abstract}
Purpose: To determine whether intrathecal administration of cultured autologous bone marrow stromal cells (BMSCs) is safe and feasible for treatment of subacute spinal injury.

Methods: Five patients with complete tetraplegia due to cervical spinal injury on admission were included. A small amount of bone marrow was obtained during surgery for spinal fusion. BMSCs were cultured, reaching $10^{7}-10^{8}$ cells. The properties and functional efficacy of the BMSCs were verified with surface marker analysis and a neurite extension test. BMSCs were administered by lumbar puncture. The patients were closely observed for 6 months, and the Committee on Effectiveness and Safety of Clinical Treatment (CESCT) evaluated safety.

Results: No adverse responses were observed in biochemical and radiographic examinations. The CESCT did not recognize any harmful effects of the transplantation, and concluded it was safe for treatment. The patients were further followed up for 1 to 4 years with no adverse responses. The recovery of American Spinal Injury Association Impairment Scale (AIS) B and C patients at transplantation was rapid and remarkable, but gradual or limited in AIS A patients.

Conclusion: This study demonstrated that intrathecal administration of cultured autologous BMSCs is safe and feasible for treatment of spinal cord injury.
\end{abstract}

Keywords: Spinal cord injury treatment, bone marrow stromal cell, spinal cord regeneration, cerebrospinal fluid

\section{Introduction}

Spinal cord injury (SCI) with loss of motor and sensory function often results in devastating dysfunction

*Corresponding author: Toshio Nakatani, MD, Department of Emergency and Critical Care Medicine, Kansai Medical University, 10-15, Fumizono-cho, Moriguchi, 570-8507 Osaka, Japan. Tel.:/Fax: +81 66993 9530; E-mail: nakatant@takii.kmu.ac.jp. and disability. The number of patients with SCI is estimated at over 2 million worldwide (Fawcett et al., 2007). They suffer from complications such as decubitus, infection of the urinary and respiratory tracts and so on. After studies of degenerative mechanisms (Hagg and Oudega, 2006) and clinical recovery courses (Geisler et al., 2001), new procedures with various bioactive agents (Young, 2002; Vitellaro-Zuccarello et al., 2007; Ha et al., 2005), neurotrophic factors 
(Ohori et al., 2006), transplantation of bone marrow cells (Ohta et al, 2004; Ide et al., 2010; Grigoriadis et al., 2011) and other tissues including olfactory ensheathing cells (Huang et al., 2009) are being investigated for their effects either on inhibiting secondary damage or enhancing regeneration of the neural cells.

In a previous study, we reported that bone marrow stromal cells (BMSCs) enhanced differentiation of cocultured neurosphere cells in vitro and promoted regeneration of injured spinal cords in rats (Wu et al., 2003). We also reported that administration of BMSCs into cerebrospinal fluid (CSF) promoted functional recovery of rats with SCI (Ohta et al., 2004). Recently, 6 out of 10 SCI dogs treated with intrathecal administration of autologous BMSCs showed significant motor improvement compared with untreated SCI dogs (Nishida et al., 2010). We think that BMSCs are the most appropriate cells for use in the clinical treatment of SCI. After repeated discussion with SCI patient groups, we started an SCI treatment clinical trial using the least invasive method for intrathecal administration of cultured autologous BMSCs in 2005. Our approach was to use lumbar puncture to transplant BMSCs into subacute phase victims with the approval of the Ethics Review Boards of our institutions. In the present study, we included five cervical SCI cases that met the eligibility criteria of the clinical trial. No adverse effects have been observed up to 1 year (case \#5) to 4 (case \#1).

\section{Materials and methods}

\subsection{Outline of the protocol}

The protocol of this trial was developed in accordance with the Japanese Guidelines for Clinical Studies issued in July 2003 by the Ministry of Health Welfare and Labor, and was approved by the Ethics Review Board of our institutions. This clinical trial has been registered at www.clinicaltrials.gov with identifier No. NCT00695149.

Patients with severe spinal cord injury who needed surgery for spinal stabilization with a bone graft in the acute phase (within $72 \mathrm{~h}$ after the injury) were regarded as eligible for this study. Eligibility and exclusion criteria of injured patients are shown in Tables 1 and 2 (Saito et al., 2008). During the surgery, iliac cancellous bone of ca. $5 \mathrm{~cm}^{3}$ was obtained, and BMSCs were cultured and proliferated as described below. The cell properties
Table 1

Eligibility criteria for preliminary registration

1) Spinal cord injury is confirmed by MRI

2) AIS is A, B or C

3) ISCSCI motor function score can be evaluated

4) Methylprednisolone therapy according to the NASCIS II study can be started within 8 hours after the injury

5) BMSCs incubation can be started within $72 \mathrm{hrs}$ after the injury

6) Age from 15-60

7) With the first informed consent to obtain bone marrow

MRI = magnetic resonance imaging

AIS = American Spinal Injury Association Impairment Scale.

(A = Sensorimotor complete lesion. No motor or sensory function preserved in sacral segments.

$\mathrm{B}=$ Motor complete lesion. Sensory but not motor function preserved in at least the sacral segments.

$\mathrm{C}=$ Motor and sensory incomplete lesion. Some motor function preserved below the neurologic level, but more than half of the key muscles involved have a muscle strength score $<3$.

$\mathrm{D}=$ Motor and sensory incomplete lesion. Motor function preserved below the neurologic level but more than half of the key muscles have a muscle grade of 3 or more).

ISCSCI = International Standard Classification of Spinal Cord Injury.

NASCIS = National Acute Spinal Cord Injury Study.

$\mathrm{BMSC}=$ Bone Marrow Stromal Cell.

Cited from Saito F, Nakatani T, et al: Spinal cord injury treatment with intrathecal autologous bone marrow stromal cell transplantation. J Trauma 64(1): 53-59, 2008, with LWW Journal Permissions License Number 2481700484205.

Table 2

Exclusion criteria

1) complete disruption of spinal cord

2) central spinal cord injury

3) spinal canal stenosis before the injury

4) brain or spinal cord disease before the injury

5) multiple organ disease of SOFA score $\geq 12$

6) multiple trauma victim with injuries abbreviated injury score $\geq 2$ in more than 2 segments except for the spinal injury

7) positive serological test in at least one of the following; HBs antigen, HCV antibody, HIV antibody, or HTLV-1 antibody

8) pregnancy

SOFA = Sepsis-related Organ Failure Assessment.

$\mathrm{HBs}=$ Hepatitis B surface antigen.

$\mathrm{HCV}=$ Hepatitis C Virus.

HIV = Human Immunodeficiency Virus.

HTLV = Human T-cell Leukemia Virus.

Cited from Saito F, Nakatani T, et al: Spinal cord injury treatment with intrathecal autologous bone marrow stromal cell transplantation. J Trauma 64(1): 53-59, 2008 with LWW Journal Permissions License Number 2481700484205.

and functional efficacy were examined before transplantation in the last 3 cases (\#3, \#4, and \#5). BMSCs were administered into the CSF by lumbar puncture 
within 3 weeks post injury. The aim of this trial was to determine the safety and feasibility of intrathecal administration of cultured autologous BMSC.

\subsection{Initial treatments}

When patients with SCI were admitted to our hospital, they were treated with airway management, respiratory-assisted or mechanical-ventilated tracheal intubation, fluid resuscitation with or without dopamine administration, and cervical stabilization with a neck collar. X-ray and CT examinations were followed by emergency magnetic resonance imaging (MRI). When SCI was diagnosed and the patient was not contraindicated for the methylprednisolone protocol, a single bolus injection of $30 \mathrm{mg} / \mathrm{kg}$ methylprednisolone was administered within 8 hours after the injury followed by continuous administration of $5.4 \mathrm{mg} / \mathrm{kg} / \mathrm{hr}$ for 23 hours according to the National Spinal Cord Injury Study II (Bracken et al., 1990). On the day of admission, the patient underwent installation of a Halo Brace to prevent secondary injury due to instability of the cervical spine. Neurologic functional classification was evaluated according to the American Spinal Injury Association (ASIA) Impairment Scale (AIS) grades A-E. ASIA motor score was calculated according to the International Standards for Neurological and Functional Classification of Spinal Cord Injury. When the patient met the eligibility criteria but not those for exclusion, we informed the patient's spouse or his guardian of this clinical trial. We explained the whole process of the clinical trial to them. The patient and/or family/guardian were all upset at the time of admission immediately after the injury, so it was difficult to obtain written consent for the whole process of the clinical trial. Therefore, we obtained written consent in two steps: the first consent was only to collect cancellous bone from the iliac crest for BMSC culture during the cervical stabilization surgery. Since this clinical study was indicated exclusively for patients who received bone graft surgery from the iliac crest for spinal stabilization with or without instrumentation, surgery to obtain a small amount of cancellous bone from the iliac crest for BMSC culture posed a minimal additional insult to the patient. Several days after the first written consent, when the patient and his or her family members had calmed down, we discussed entry to the BMSC transplantation clinical trial. If they accepted inclusion, we obtained written consent for the clinical trial. Decompression surgery was performed immediately after admission to the hospital or at the latest within 3 days post-injury, if needed.

\subsection{Cell culture}

\subsubsection{Cell culture}

Cancellous bone fragments (ca. $5 \mathrm{~cm}^{3}$ ) with bone marrow tissue were obtained from the iliac crest of each patient during surgery. In a cell-processing center that met the cell culture guideline for good manufacturing practice for clinical treatment in Japan, cancellous bone was cut and triturated to dissolve bone marrow cells in $\alpha$-minimum essential medium (MEM) (Invitrogen, Carlsbad, CA, USA). The cell suspension was filtered through a $100-\mu \mathrm{m}$ nylon mesh cell strainer, centrifuged, and suspended in a 40-ml serumcontaining the culture medium comprising $\alpha$-MEM supplemented with $10 \%$ fetal bovine serum (FBS) (Invitrogen, Carlsbad, CA, USA). The cell suspension was seeded into 10-15 flasks (T-75, Becton Dickinson, Franklin Lakes, NJ, USA), and cultured at $37^{\circ} \mathrm{C}$ in a $5 \% \mathrm{CO}_{2}$ in air atmosphere. FBS was obtained from New Zealand, a country free of bovine spongioform encephalopathy. BMSCs adherent to the flask grew to confluence within 5-7 days after cell plating. The adherent cells which had proliferated to confluence were dissociated for passaging into many flasks to allow further proliferation. One flask contained approximately $3-5 \times 10^{6}$ cells in a confluent condition. Accordingly, at least 10-15 flasks containing confluent cells were needed for cell transplantation. One cell passage was enough for an adequate cell population in 4 cases, and no cell passage was needed in one case. Before transplantation, adherent BMSCs were dissociated and suspended in 2-4 $\mathrm{ml}$ of saline for transplantation.

To check cell viability, an aliquot $(40 \mu \mathrm{l})$ of suspension was obtained and mixed with an equal volume of trypan-blue solution (Invitrogen, Carlsbad, CA, USA). Viable cells (dye-excluding cells) were counted on a hemocytometer.

\subsubsection{Evaluation of cell properties}

Prior to cell transplantation, cell properties were evaluated by immunohistochemistry and in vitroneurite extension assessment. For immunohistochemical assessment, a small volume $(2 \mathrm{ml})$ of the primary cell suspension from cancellous bones was cultured separately in 2 eight-chamber slides for 7-10 days, as described above. For immunostaining, cells adhering 
to the chamber were fixed in $2 \%$ paraformaldehyde in $0.1 \mathrm{M}$ phosphate buffer. After washing in PBS, cells were incubated with primary antibodies for CD90, CD11b (these two, PharMingen, San Diego, CA, USA), and CD34 (Santa Cruz Biotechnology, Santa Cruz, CA, USA) for 24 hours at $4{ }^{\circ} \mathrm{C}$. Two chambers were used for the immunostaining with each antibody. After washing, cells were stained with FITC (Fluorescein isothiocyanate)-conjugated secondary antibodies (Amersham, Buckinghamshire, UK) for 1 hour, and observed with a fluorescence microscope (Axio Imager MI, Carl Zeiss, Goettingen, Germany). Cell nuclei were stained with 4, 6-diamidino-2-phenylindole (DAPI) to calculate the total cell number.

We tentatively provided criteria for human BMSCs to be used for transplantation as follows: $\mathrm{CD} 90^{+}$cells should be more than $30 \%$, while CD34 ${ }^{+}$and CD $11 b^{+}$ cells should be less than $10 \%$.

For in vitro-neurite extension assessment, a small volume $(2 \mathrm{ml})$ of the primary cell suspension from cancellous bones with addition of an equal volume of fresh culture medium, seeded in 10 wells of 48-well microplates, and cultured for 7-10 days, as described above. The adherent cells grew to confluence at 7 to 10 days of culture. After washing with PBS, cells were cultured for 24 hours in a medium containing fresh $\alpha$ MEM without FBS to obtain serum-free conditioned medium (CM). This serum-free $\mathrm{CM}$ was used to assess neurite extension of a hippocampal neuron culture as described below.

Hippocampal neurons were cultured as follows. The hippocampus was excised from postnatal day 1-2 Spraque-Dawley rats (SLC, Shizuoka, Japan), and dissociated in Nerve Cell Dissociation Solution CP (DS Pharma Biomedical, Suita, Osaka, Japan) according to the manufacturer's instructions. Neurons were seeded on 10 poly-L-lysine (PLL)-coated wells of 48-well microplates, and cultured in Neurobasal medium containing B27 supplement (Invitrogen, Carlsbad, CA, USA), $2 \mathrm{mM}$ L-glutamine, penicillin, and streptomycin (NB/B27 medium).

For the neurite extension assay, after removal of NB/B27 medium, cultured neurons were re-fed with the CM obtained from the 24-hour culture of BMSCs as described above, and cultured for 24 hours. Extending neurites more than $20 \mu \mathrm{m}$ long were regarded as neurons with neurites. The eligible condition for transplantation was that neurons with neurites should comprise more than $70 \%$ of the total neurons in more
Table 3

Eligibility criteria for full registration

1) $>10^{6}$ BMSCs are obtained by tissue culture

2) Transplantation can be performed $<3$ weeks after the injury

3) AIS A, B or C grade is confirmed within 3 days prior to transplantation

4) Second informed consent for transplantation is obtained

$\mathrm{BMSC}=$ Bone Marrow Stromal Cell.

AIS = American Spinal Injury Association Impairment Scale. Cited from Saito F, Nakatani T, et al: Spinal cord injury treatment with intrathecal autologous bone marrow stromal cell transplantation. J Trauma 64(1): 53-59, 2008, with LWW Journal Permissions License Number 2481700484205.

than 5 visual fields of a phase-contrast microscope (Olympus, Tokyo, Japan).

\subsection{Transplantation}

During the incubation period, we had enough time to inform families and patients again of clinical trial details. After obtaining the second written consent to the clinical trial from the patient and family, we confirmed the final eligibility criteria (Table 3) (Saito et al., 2008). Each clinical trial including cell transplantation was discussed and approved by the Ethics Review Board.

On the morning of transplantation, BMSCs were dissociated from flasks; $3-5 \times 10^{7}$ BMSCs were suspended in about $2 \mathrm{~mL}$ of saline as described above. The suspension drawn in a syringe was transported to the hospital and transplanted through the L3-L4 inter-vertebral space into the CSF by lumbar puncture. BMSCs should be transplanted within 4 hours after dissociation. The transplantation was performed with minimal invasive insult with only a single injection. No surgical procedure was necessary to re-open and divide the spinal cord wound made during the decompression surgery 1-2 weeks earlier.

According to the protocol, the patients were observed until 6 months after cell transplantation, during which AIS grade and ASIA motor score were evaluated at 3 and 6 months. CT and MRI of the spine and spinal cord were also taken at 3 and 6 months. To date, they have been followed up for 1 to 4 years. One month or more following cell transplantation, the CESCT plus some neutral members from outside this study group discussed the safety of each case, and if the safety was acceptable, it was agreed that we could proceed to the next case trial. 
Table 4

Patient information and changes in the AIS and ASIA motor scores

\begin{tabular}{|c|c|c|c|c|c|c|c|c|c|c|}
\hline \multirow[t]{2}{*}{ No. } & \multirow[t]{2}{*}{ Age } & \multirow[t]{2}{*}{ Injury } & \multirow{2}{*}{$\begin{array}{c}\text { Day } \\
\text { of TX }\end{array}$} & \multicolumn{2}{|c|}{ Size of SCI $(\mathrm{mm})$} & \multicolumn{2}{|c|}{ AIS } & \multicolumn{3}{|c|}{ Motor score } \\
\hline & & & & Initial & $6 \mathrm{M}$ & Initial & $6 \mathrm{M}$ & Initial & $3 \mathrm{M}$ & $6 \mathrm{M}$ \\
\hline 1 & 35 & $\mathrm{C} 5: \mathrm{DL}+\mathrm{FX}$ & 13 & $62 \times 8$ & $21 \times 11$ & A & A & 6 & 16 & 17 \\
\hline 2 & 59 & C6: DL & 8 & $23 \times 6$ & $5 \times 5$ & B & $\mathrm{D}$ & 5 & 59 & 79 \\
\hline 3 & 45 & $\mathrm{C} 4: \mathrm{DL}+\mathrm{FX}$ & 13 & $38 \times 6$ & $11 \times 6$ & $C^{* *}$ & $\mathrm{D}$ & 13 & 60 & 72 \\
\hline 4 & 23 & $\mathrm{C} 5: \mathrm{DL}+\mathrm{FX}$ & 17 & ND & $70 \times 22 *$ & A & A & 7 & 8 & 8 \\
\hline 5 & 51 & $C 4-6: D L+F$ & 14 & $65 \times 9$ & $45 \times 15$ & A & A & 3 & 3 & 3 \\
\hline
\end{tabular}

All the five patients were male.

AIS = American Spinal Injury Association Impairment Scale. $(A=$ Sensorimotor complete lesion. No motor or sensory function preserved in sacral segments. $\mathrm{B}=$ Motor complete lesion. Sensory but not motor function preserved in at least the sacral segments. $\mathrm{C}=\mathrm{Motor}$ and sensory incomplete lesion. Some motor function preserved below the neurologic level, but more than half of the key muscles involved have a muscle strength score $<3$. $\mathrm{D}=$ Motor and sensory incomplete lesion. Motor function preserved below the neurologic level but more than half of the key muscles have a muscle grade of 3 or more).

$\mathrm{SCI}=$ spinal cord injury. $\mathrm{TX}=$ transplantation.

$\mathrm{DL}=$ dislocation, $\mathrm{FX}=$ fracture, $\mathrm{ND}=$ near disruption.

*size of cerebrospinal fluid collection.

**at the time of cell transplantation.

\section{Results}

We completed the clinical trial for each case with at least a 6-month follow-up as documented in the protocol for the 5 patients. All 5 cases had cervical spinal injury with dislocation and/or fracture of the spine. According to the protocol, only those patients who had bone graft surgery for spinal fusion were indicated for this clinical trial. The patients' information and the AIS and ASIA motor score data before surgery and up to 6 months after the transplantation are summarized in Table 4.

Table 5 shows the results of cell cultures. Examination of cell surface markers and neurite extension tests were performed before transplantation in the latter 2 cases (cases \#4 and \#5) and are shown in the text below. All the cultured cells were confirmed to be free from mycoplasma, endotoxins $(<12 \mathrm{EU})$ and bacteria before transplantation.

As neurological examinations are unstable shortly after spinal injury, AIS and ASIA motor scores at the

Table 5

Cell culture results

\begin{tabular}{lccccc}
\hline$\#$ & $\begin{array}{c}\text { Cancellous } \\
\text { bone obtained }\end{array}$ & $\begin{array}{c}\text { Culture } \\
\text { period }\end{array}$ & $\begin{array}{c}\text { Number } \\
\text { of passes }\end{array}$ & $\begin{array}{c}\text { Number of } \\
\text { cells obtained }\end{array}$ & $\begin{array}{c}\text { Cell } \\
\text { viability }\end{array}$ \\
\hline 1 & $3 \mathrm{~cm}^{3}$ & 10 days & 1 & $3.1 \times 10^{7}$ & $84.1 \%$ \\
2 & 5 & 8 & 0 & 2.1 & 91.7 \\
3 & 4 & 12 & 1 & 3.7 & 94.0 \\
4 & 3 & 15 & 1 & 10.0 & 92.0 \\
5 & 5 & 11 & 1 & 10.0 & 98.0 \\
\hline
\end{tabular}

Cell culture and viability tests were performed as stated in the text. pre-transplantation stage were assessed 1-2 weeks post injury. On MRI, the contusion area exhibited in the first three cases as a small-sized cavity formation at 3 and 6 months (Fig. 1), while in cases \#4 and \#5, the contusion area showed a large space of CSF accumulation occupying the whole vertical section of the injured spinal cord (Figs. 2, 3).

Case \#1 (male, 35-year-old) had a spinal cord injury at the $\mathrm{C} 5$ level after falling from height on March 11, 2006. C5 motor function was partially preserved and he was able to minimally flex his elbow joints, but no motor function was observed below the level of C6 on day 13. The patient showed elevated serum aspartate aminotransferase (AST), alanine aminotransferase

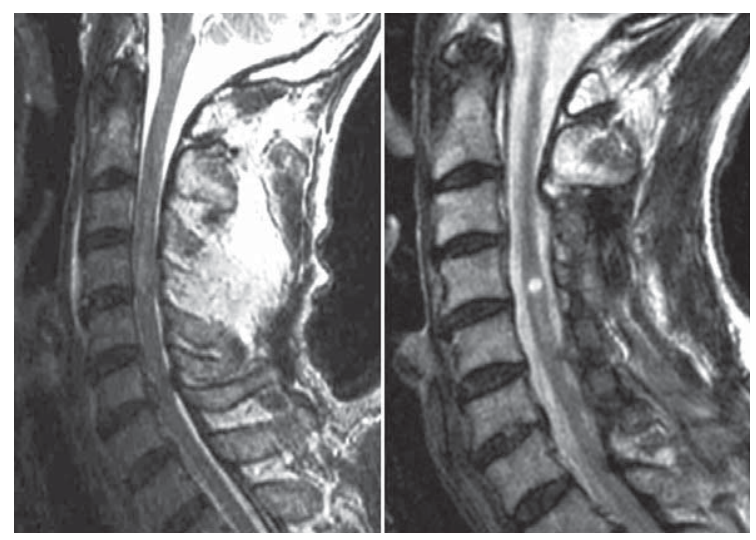

Fig. 1. MRI of case \#2. T2-weighted image showed a high intensity area at the level of C3-4 on admission (left), but became smaller at 6 months (right). 


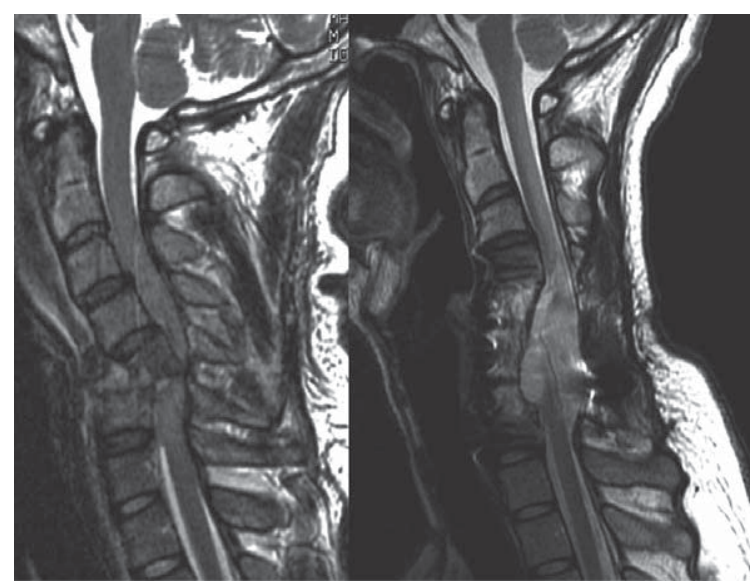

Fig. 2. MRI of case \#4. Spinal cord was nearly disrupted due to C5 spine fracture and dislocation on admission (left), CSF collection at 6 months (right).

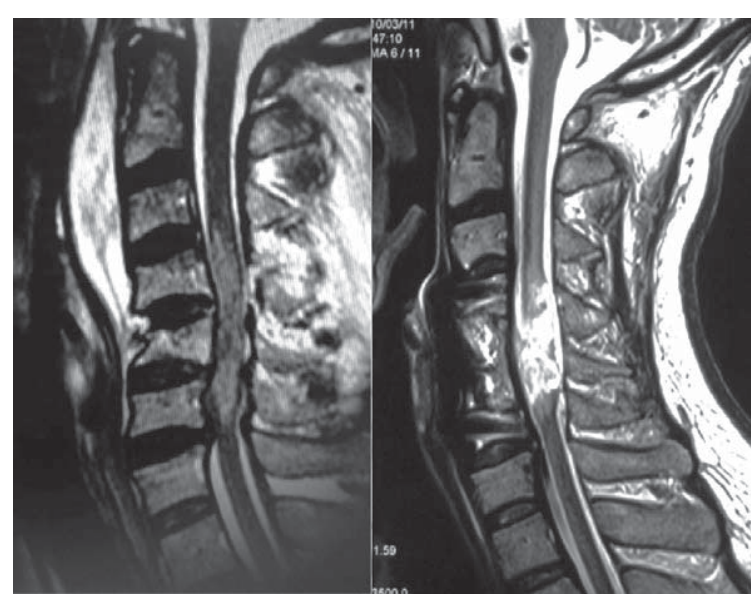

Fig. 3. MRI of case \#5. C4-6 spinal cord was widely crushed on admission (left). At 6 months, the spinal cord was swollen and mixed with hematoma (right). The size of the spinal swelling was unchanged compared with that at 3 months.

(ALT), creatine kinase (CK), lactate dehydrogenase (LDH), and amylase levels, and reduced hemoglobin, albumin, $\mathrm{Na}$ and $\mathrm{Cl}$ levels. All these changes had begun before the transplantation and continued for a short time after cell transplantation. He suffered from fever and leucocytosis due to a urinary tract infection a few weeks after cell transplantation. He also suffered from temporal atelectasis due to motor paralysis of respiratory muscles. The CESCT discussed these examination data, and concluded that these were not adverse reactions related to the intrathecal cell administration. Three months after cell transplantation, he could maintain a sitting position and drive a wheel chair by himself. There was no abnormal change in MRI during the follow-up period.

Case \#2 (male, 59-year-old) suffered a spinal cord injury at C6 after falling down some stairs on March 23,2008 . As in case \#1, he was able to minimally flex his elbow joints, but no motor function was observed below the level of C6 on day 8. Although motor function loss was complete, slight sensory function remained below the C6 level. Therefore, this SCI was classified as AIS grade B at the time of transplantation. He showed slight AST and ALT elevation before transplantation, which continued for a while afterwards. No other abnormal examination data were found. Seven days after the transplantation, his motor function began to improve rapidly and markedly. He could stand up and walk slowly without assistance at 3 months, and stand up and walk around without any help at 6 months after transplantation. The MRI finding showed no change at 3 and 6 months post-transplantation, and there has been no abnormal change in the follow-up period (Fig. 1).

Case \#3 (male, 45-year-old) suffered a spinal cord injury at $\mathrm{C} 4$ after falling down some stairs on May 21,2009 . He showed sensorimotor complete paralysis without a sacral sparing effect, and was classified as AIS grade A. After surgery for decompression and stabilization with instrumentation, the sensorimotor lesion remained unchanged for more than a week. However, functional assessment just before transplantation showed a slight recovery of anal sphincter function, while complete sensory loss below $\mathrm{C} 4$ continued at that time. The AIS grade was classified as $\mathrm{C}$ before transplantation.

He showed mild elevation of ALT shortly after surgery, which returned to the normal level shortly after cell transplantation. These events were not considered to be an adverse response. Cell surface marker examinations were done for this case. The data were as follows; CD11b 2.1\%, CD34 5.5\%, and CD90 87.8\%. Neurite extension assessment was positive for $82.4 \%$ of neurons. MRI findings showed no change at 3 and 6 months post-transplantation, and there has been no abnormal change in the follow-up period.

After transplantation, motor function recovered in the lower extremities, although sensory function remained mostly dull or disappeared. His leg muscles were spastic and sensory function was lost, and although he was able to stand using equipment, he could not walk 6 months after cell transplantation. 
Case \#4 (male, 23-year-old) was a victim of a traffic accident. He suffered a spinal cord injury at $\mathrm{C} 4$ on July 5, 2009. The spinal cord was nearly completely disrupted on imaging studies and was rated AIS grade A. The cell surface marker data were as follows: CD11b 3.7\%, CD34 3.0\%, and CD90 96.2\%. Neurite extension assessment was positive for $82.4 \%$ of neurons. Some abnormalities were found in blood chemical tests before transplantation, and some symptoms due to urinary tract infection were observed after transplantation. The CESCT concluded that none of these events were adverse responses related to cell transplantation. He has so far shown no improvement in motor function. A space of CSF accumulation was observed at the injury site at 3 months posttransplantation. It remained the same size during in the follow-up for 6 months (Fig. 2). MRI showed no proliferation sign by gadrinium examination at further follow-up. Although the patient showed no changes in motor function until 6 months post-transplantation, he was able to drive a wheel-chair 12 months after transplantation.

Case \#5 (male, 51-year-old) sustained a wide crush injury of the spinal cord at C4-6 following a fall from a rock on September 5, 2009. Cell surface marker data were as follows: CD11b 3.8\%, CD34 2.0\%, and CD90 $97.2 \%$. Neurite extension assessment was positive for $96.4 \%$ of neurons. The blood test showed some abnormalities such as leucocytosis and CRP elevation before cell transplantation, which continued for a while afterwards. The CESCT considered that these events were due to pneumonia and a urinary infection, concluding that there was no adverse response related to cell administration. On MRI, the contusion area showed a large space of CSF accumulation occupying the whole spinal cord. It remained the same size during in the follow-up for 6 months (Fig. 3).

\section{Discussion}

SCI with complete motor paralysis leads to poor results, particularly for those with tetraplegia. Treatments for such victims are very difficult and controversial. As there is no definitive effective treatment, management of patients has focused on preventing secondary damage and maintaining or maximally restoring the preserved function of the spinal cord with rehabilitation. We showed significant recovery of motor function in rats with experimental thoracic spinal cord contusion injury by transplantation of BMSCs through the CSF (Ohta et al., 2004). For the clinical application of BMSC transplantation, we used autologous BMSCs to avoid ethical and immunological problems as have other clinical trials (Attar et al., 2011; Chernykh et al., 2007; Deda et al., 2008; Geffner et al., 2008; Kishk et al., 2010; Kumar et al., 2009; Pal et al., 2009). In our protocol, we planned to include only those patients who needed surgery for cervical spinal fusion with iliac bone grafting. Iliac cancellous bones were obtained for cell culture during surgery with no additional insult (Saito et al., 2008). Bone marrow tissue was cultured for 1 or 2 weeks to allow proliferation of an adequate population (approximately $10^{6}$ cells) of BMSCs.

Clinical transplantation studies by other investigators reported a laminectomy followed by an opening and detachment of the adherent dura mater for the injection of cells into exposed spinal cord (Attar et al., 2011; Chernykh et al., 2007; Deda et al., 2008; Féron et al., 2005; Geffner et al., 2008; Huang et al., 2009; Lima et al., 2006; Mackay-Sim et al., 2008; Yoon et al., 2007). Although most authors reported the technique was safe, such surgery may damage the remaining intact tissues of the spinal cord. As a result, a few complications resulted after the surgery (Lima et al., 2006). It is crucial in cell transplantation to avoid damage to the preserved function of the spinal cord. Cell transplantation through intra-arterial or intra-venous procedures has been reported to avoid further additional damage to the injured spinal cord (Syková et al., 2006). We have shown that cell transplantation through CSF is effective for the treatment of spinal cord injury in rats (Bai et al., 2003; Ohta et al., 2004; Wu et al., 2002). Based on these experimental outcomes and a safety test using rhesus monkeys, we aimed to treat clinical SCI cases by transplantation of cultured autologous BMSCs into the CSF via lumbar puncture at the early subacute phase of SCI. We developed a detailed protocol for the clinical trial after discussions with SCI patient groups. The Ethics Review Board of both Kansai Medical University and the Translational Research Center for Cell Processing officially approved the protocol. This phase 1-2, open label non-randomized clinical trial aimed to treat a damaged spinal cord by the least invasive method of administering cultured BMSCs into the CSF via lumbar puncture (Saito et al., 2009), and assessed the feasibility and safety of the procedure. Recently, other investigators also reported the safety of the intrathecal administration by lumbar 
puncture (Pal et al., 2009; Kumar et al., 2009). We have summarized the first five cases treated under this protocol.

The cell culture was described in detail in Materials and Methods to make clear the properties of the cells used for transplantation. As described above, cell passage was done at most once before transplantation. This means that cells underwent minimum functional changes including gene expression before transplantation. Sykova et al. and Kumar et al. reported administration of bone marrow derived mononuclear cells without incubation (Kumar et al., 2009; Sykova et al., 2006). CD34 and CD11b were regarded as markers for endothelial cells and macrophages, respectively. Few cells were positive for CD34 or CD11b. On the other hand, CD90 (thy-1) is generally regarded as a marker of stromal cells. CD90-positive cells accounted for actually more than $80-90 \%$ of the cell population. This meant that we were dealing with a cell population composed almost exclusively of bone marrow stromal cells.

The autologous BMSCs used in the present study pose no ethical or immunologic problems. On MRI and CT, there were no findings of calcification possibly caused by bone marrow cells or teratoma formation possibly caused by stem cells. Other investigators also report the safety of intrathecal administration of bone marrow cells (Geffner et al., 2008; Kumar et al., 2009; Pal et al., 2009), but Kishk et al. reported spasticity and neuropathic pain in half of their patients after repeated intrathecal administration once a month for 6 months (Kishk et al., 2010). In the MRIs of cases \#4 and \#5, a space of apparent CSF collection formed within the spinal cord, presumably with hematoma around the damaged spinal cord at 3 and 6 months. However, there were no adverse responses related to this spinal cord finding or any expanding changes in images at 6-month to 1-year follow-ups. The CESCT concluded the local CSF collection, presumably with a hematoma around the damaged spinal cord is not undesirable or harmful for the spinal cord. Chernykh et al. administered bone marrow cells into the spinal cyst, and reported there were no allergic reaction, no infection and no ossification (Chernykh et al., 2007)

According to the animal experiments, a small number of BMSCs infused through the CSF attached to the injured spinal cord surface, with a few of them further invading the lesion. However, the grafted BMSCs disappeared from the spinal cord within 3 weeks after grafting (Ohta et al., 2004). BMSCs transplanted directly into the lesion also disappeared in 2 weeks (Ide et al., 2010). This suggests that grafted BMSCs may have dual functions; the release of trophic factors and induction of tissue matrix formation (Ide et al., 2010). The fact that grafted BMSCs disappeared from the spinal cord suggests that they do not cause any harmful effects including tumor formation related to stem cells in the spinal cord. Although the fate of transplanted BMSCs could not be confirmed in the clinical trial, it is natural to consider that BMSCs administered by lumbar puncture may not survive for a long time in CNS patients. We consider that the transplantation of cultured autologous BMSCs via lumbar puncture is a safe and feasible for the treatment of spinal cord injury. There are clinical studies administering bone marrow cells or olfactory ensheathing cells (OEC) via various routes. Cells are administered into the injured spinal cord (Attar et al., 2011; Deda et al., 2008; Féron et al., 2005; Geffner et al., 2008; Huang et al., 2009; Knoller et al., 2005; Lima et al., 2006; Mackay-Sim et al., 2008; Yoon et al., 2007), in the spinal cyst (Chernykh et al., 2007), into the CSF (Geffner et al., 2008; Kishk et al., 2010; Kumar et al., 2009; Pal et al., 2009), or into the systemic circulation (Geffner et al., 2008; Syková et al., 2006), but no serious side effects such as tumor formation or ossification have been reported, except for minor infection or meningitis after direct injection with laminectomy (Dobkin et al., 2006; Knoller et al., 2005).

The International Campaign for Cures of Spinal Cord Injury Paralysis (ICCP) recently supported an international panel tasked with reviewing the methodology for clinical trials in SCI, and made recommendations on the conduct of clinical trials (Fawcett et al., 2007). It has been known that spinal function varies in the acute phase immediately after injury, and many SCI patients show some recovery of spinal function below the initial spinal injury level. Spontaneous recovery of motor function in patients with complete SCI (AIS A) is fairly limited, however, recovery in incomplete SCI patients (AIS C and AIS D) is both more substantial and highly variable. It is not uncommon that AIS B patients recover to AIS D. Some trials reported a high rate of recovery after OEC (Huang et al., 2009) or bone marrow cell administration (Deda et al., 2008), and others report minor or minimal improvements (Geffner et al., 2008; Kumar et al., 2009; Mackay-Sim et al., 2008; Pal et al., 2009; Yoon et al., 2007), but it is difficult to evaluate the effectiveness without a control group. In the clinical studies 
compared with an untreated control group, only minor motor score improvements were reported (Chernykh et al., 2007; Kishk et al., 2010). As the nature of spinal injury is pessimistic, it will be difficult to complete a double-blinded case control study. Recently, in the field of veterinary medicine, 10 dogs with accidental chronic spinal injury were treated with our methods and exhibited significantly improved motor function compared with 13 untreated injured dogs (Nishida et al., 2010)

It has reported that the rate of recovery is rapid during the first three months, and motor improvement is almost complete by 9 months, but plateaus later than 12 months (Fawcett et al., 2007). The motor recovery measured in ASIA motor scores over the first year after the SCI in cervical AIS B patients are reported to be 30 to 40 points in large patient number studies (Fawcett et al., 2007; Waters et al., 1993). Compared with results of Fawcett et al., 2007, the recovery of motor functions after cell transplantation is gradual but definite in case $\# 1$, and rapid and remarkable in cases of \#2 and \#3, even though it was limited in other ASIA A patients.

We consider that the clinical trial by BMSC transplantation via lumbar puncture in the present study is safe and feasible. Concerning the effectiveness of BMSC transplantation, the data presented here suggest that BMSC transplantation can be highly effective and beneficial for the treatment of spinal cord injury.

\section{Acknowledgments}

This work was supported, in part, by a Grant-inAid for Scientific Research from Japan Society for the Promotion of Science, Japan. Grant number: No. 18390482 for Toshio Nakatani.

\section{References}

Attar, A., Ayten, M., Ozdemir, M., et al. (2011). An attempt to treat patients who have injured spinal cords with intralesional implantation of concentrated autologous bone marrow cells. Cytotherapy, 13, 54-60.

Bai, H., Suzuki, Y., Noda, T., et al. (2003). Dissemination and proliferation of neural stem cells on the spinal cord by injection into the fourth ventricle of the rat: A method for cell transplantation. J Neurosci Methods, 124, 181-187.

Bracken, M.B., Shepard, M.J., Collins, W.F., et al. (1990). A randomized, controlled trial of methylprednisolone or naloxone in the treatment of acute spinal-cord injury. $N$ Engl J Med, 322, 1405-1411.
Chernykh, E.R., Stupak, V.V., Muradov, G.M., et al. (2007). Application of autologous bone marrow stem cells in the therapy of spinal cord injury patients. Bull Exp Biol Med, 143, 543-547.

Deda, H., Inci, M.C., Kurekci, A.E., et al. (2008). Treatment of chronic spinal cord injured patients with autologous bone marrow-derived hematopoietic stem cell transplantation: 1-year follow-up. Cytotherapy, 10, 565-574.

Dobkin, B.H., Curt, A. \& Guest, J. (2006). Cellular transplants in China: Observational study from the largest human experiment in chronic spinal cord injury. Neurorehabil Neural Repair, 20 , $5-13$.

Fawcett, J.W., Curt, A., Steeves, J.D., et al. (2007). Guidelines for the conduct of clinical trials for spinal cord injury as developed by the ICCP panel: Spontaneous recovery after spinal cord injury and statistical power needed for therapeutic clinical trials. Spinal Cord, 45, 190-205.

Féron, F., Perry, C., Cochrane, J., et al. (2005). Autologous olfactory ensheathing cell transplantation in human spinal cord injury. Brain, 128, 2951-2960.

Geisler, F.H., Coleman, W.P., Grieco, G., et al. (2001). Measurements and recovery patterns in a multicenter study of acute spinal cord injury. Spine, 26, S68-S86.

Geffner, L.F., Santacruz, P., Izurieta, M., et al. (2008). Administration of autologous bone marrow stem cells into spinal cord injury patients via multiple routes is safe and improves their quality of life: Comprehensive case studies. Cell Transplant, 17, 1277-1293

Grigoriadis, N., Lourbopoulos, A., Lagoudaki, R., et al. (2011). Variable behavior and complications of autologous bone marrow mesenchymal stem cells transplanted in experimental autoimmune encephalomyelitis. Exp Neurol, 230, 78-89.

Ha, Y., Park, H.S., Park, C.W., et al. (2005). Granulocyte macrophage colony stimulating factor (GM-CSF) prevents apoptosis and improves functional outcome in experimental spinal cord contusion injury. Clin Neurosurg, 52, 341-347.

Hagg, T. \& Oudega, M. (2006). Degenerative and spontaneous regenerative processes after spinal cord injury. J Neurotrauma, 23, 264-280.

Huang, H., Chen, L., Xi, H., et al. (2009). Olfactory ensheathing cells transplantation for central nervous system diseases in 1,255 patients. Zhongguo Xiu Fu Chong Jian Wai Ke Za Zhi, 23, 1420.

Ide, C., Nakai, Y., Nakano, N., et al. (2010). Bone marrow stromal cell transplantation for treatment of sub-acute spinal cord injury in the rat. Brain Res, 1332, 32-47.

Kishk, N.A., Gabr, H., Hamdy, S., et al. (2010). Case control series of intrathecal autologous bone marrow mesenchymal stem cell therapy for chronic spinal cord injury. Neurorehabil Neural Repair, 24, 702-708.

Knoller, N., Auerbach, G., Fulga, V., et al. (2005). Clinical experience using incubated autologous macrophages as a treatment for complete spinal cord injury: Phase I study results. J Neurosurg Spine, 3, 173-181.

Kumar, A.A., Kumar, S.R., Narayanan, R., et al. (2009). Autologous bone marrow derived mononuclear cell therapy for spinal cord injury: A phase I/II clinical safety and primary efficacy data. Exp Clin Transplant, 7, 241-248. 
Lima, C., Escada, P., Pratas-Vital, J., et al. (2006). Olfactory mucosa autografts in human spinal cord injury: A pilot clinical study. $J$ Spin Cord Med, 29, 191-203.

Mackay-Sim, A., Féron, F., Cochrane, J., et al. (2008). Autologous olfactory ensheathing cell transplantation in human paraplegia: A 3-year clinical trial. Brain, 131, 2376-2386.

Nishida, H., Nakayama, M., Tanaka, H., et al. (2011). Evaluation of transplantation of autologous bone marrow stromal cells into the cerebrospinal fluid for treatment of chronic spinal cord injury in dogs. Am J Vet Res, 72, 1118-1123.

Ohori, Y., Yamamoto, S., Nagao, M., et al. (2006). Growth factor treatment and genetic manipulation stimulate neurogenesis and oligodendrogenesis by endogenous neural progenitors in the injured adult spinal cord. J Neurosci, 26, 11948-11960.

Ohta, M., Suzuki, Y., Noda, T., et al. (2004). Bone marrow stromal cells infused into the cerebrospinal fluid promote functional recovery of the injured rat spinal cord with reduced cavity formation. Exp Neurol, 187, 266-278.

Pal, R., Venkataramana, N.K., Bansal, A., et al. (2009). Ex vivo-expanded autologous bone marrow-derived mesenchymal stromal cells in human spinal cord injury/paraplegia: A pilot clinical study. Cytotherapy, 11, 897-911.

Saito, F., Nakatani, T., Iwase, M., et al. (2008). Spinal cord injury treatment with intrathecal autologous bone marrow stromal cell transplantation: The first clinical trial case report. J Trauma, 64, 53-59.

Syková, E., Homola, A., Mazanec, R., et al. (2006). Autologous bone marrow transplantation in patients with subacute and chronic spinal cord injury. Cell Transplantation, 15, 675-687.
Vitellaro-Zuccarello, L., Mazzetti, S., Madaschi, L., et al. (2007). Erythropoietin-mediated preservation of the white matter in rat spinal cord injury. Neuroscience, 144, 865-877.

Waters, R.L., Adkins, R.H., Yakura, J.S., et al. (1993). Motor and sensory recovery following complete tetraplegia. Arch Phys Med Rehabil, 74, 242-247.

Wu, S., Suzuki, Y., Kitada, M., et al. (2001). Migration, integration, and differentiation of hippocampus-derived neurosphere cells after transplantation into injured rat spinal cord. Neurosci Lett, $312,173-176$

Wu, S., Suzuki, Y., Kitada, M., et al. (2002). New method for transplantation of neurosphere cells into injured spinal cord through cerebrospinal fluid in rat. Neurosci Lett, 318, 81-84.

Wu, S., Suzuki, Y., Ejiri, Y., et al. (2003). Bone marrow stromal cells enhance differentiation of cocultured neurosphere cells and promote regeneration of injured spinal cord. J Neurosci Res, 72, 343-351.

Yoon, S.H., Shim, Y.S., Park, Y.H., et al. (2007). Complete spinal cord injury treatment using autologous bone marrow cell transplantation and bone marrow stimulation with granulocyte macrophage-colony stimulating factor: Phase I/II clinical trail. Stem Cells, 25, 2066-2073.

Young, W. (2002). Methylprednisolone and spinal cord injury. $J$ Neurosurg, 96(Suppl 1), 141-142. 